\title{
Alterstice
}

Revue internationale de la recherche interculturelle

International Journal of Intercultural Research

Revista International de la Investigacion Intercultural

\section{Fabriquer un numéro : des relations et du temps}

\section{Yvan Leanza}

Volume 3, numéro 1, 2013

URI : https://id.erudit.org/iderudit/1077493ar

DOI : https://doi.org/10.7202/1077493ar

Aller au sommaire du numéro

Éditeur(s)

Alterstice

ISSN

1923-919X (numérique)

Découvrir la revue

Citer ce document

Leanza, Y. (2013). Fabriquer un numéro : des relations et du temps. Alterstice, 3(1), 1-2. https://doi.org/10.7202/1077493ar d'utilisation que vous pouvez consulter en ligne. 


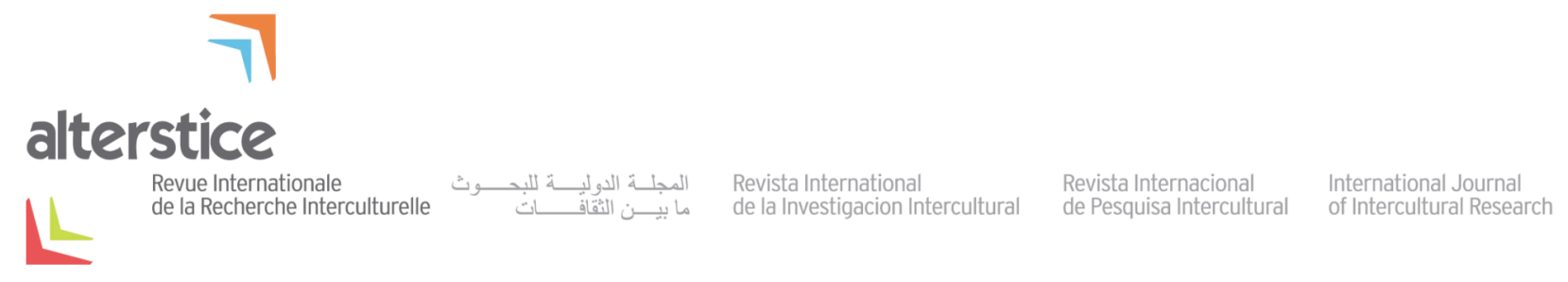

ÉDITORIAL

\section{Fabriquer un numéro : des relations et du temps}

Yvan Leanza $^{1}$

Pour son cinquième numéro, Alterstice se fait l'hôte de certaines des conférences prononcées au congrès de I'Association internationale pour la Recherche Interculturelle (ARIC) qui eut lieu à Sherbrooke (Québec, Canada) en juin 2011. Même si, a priori, un tel événement devrait assurer matière à publication aussi bien en termes de qualité que de quantité, il y a toujours des risques de se retrouver, comme organisateur du congrès puis comme éditeur d'un tel recueil, sans texte à éditer, voire avec des textes non publiables dans une revue aux critères de publications exigeants. C'est pourquoi je tiens à remercier chaleureusement Michèle Vatz Laaroussi et Claude Gélinas, aussi bien pour l'accueil et l'organisation du congrès de Sherbrooke, que pour avoir réussi à colliger les textes du présent numéro.

Fabriquer un numéro, ce n'est pas seulement cliquer sur le bouton du système informatique qui permet de mettre les textes en ligne... Cette opération est la dernière d'une longue série d'échanges, de réflexions-créations et de décisions. Du côté de l'auteur, écrire et soumettre un article est l'aboutissement du processus de recherche ou de réflexion sur un thème, souvent longuement étudié. Du côté de l'éditeur invité, il s'agit de construire une problématique rassembleuse, un objet suffisamment large et attrayant pour que les collègues s'y reconnaissent et aient envie de soumettre leur travail. II faut ensuite lire les textes soumis, trouver des évaluateurs externes, lire les évaluations, peser le pour et le contre et faire des choix pas toujours simples. Tout cela, dans le cas d'Alterstice, se fait avec l'aide de la direction de la revue, ce qui implique des échanges nombreux selon différents modes de communication (en personne, par Skype, courriel, téléphone...). Du côté de la revue, il faut s'assurer que le thème choisi par l'éditeur cadre avec les objectifs de la revue, que les évaluateurs reçoivent les bonnes informations quant aux objectifs de la revue et de son mode d'évaluation, que les temps d'évaluation, de réponses des auteurs aux demandes de modifications et de révisions soient raisonnables pour que la revue paraisse deux fois l'an. L'ensemble de ce processus implique de nombreuses personnes aux parcours et sensibilités variées, sachant que parfois les décisions quant aux changements demandés ou au refus de publication seront vécues douloureusement par les auteurs. II s'agit aussi de construire chaque numéro avec une grande diversité d'approches disciplinaires et méthodologiques et avec parfois, comme pour le présent numéro, une diversité de thèmes traitant de la diversité... 
En bref, les ingrédients fondamentaux pour la fabrication d'un numéro sont avant tout des relations et du temps. Ce temps de la recherche et de sa valorisation par la publication, que d'aucuns voudraient raccourcir pour plus de rentabilité (quelle barbarie!), est nécessaire pour saisir les réalités sociales complexes, les objectiver et les rendre accessibles aux collègues et autres personnes intéressées. Les relations, même rendues difficiles par les contraintes institutionnelles ou éditoriales ou par manque d'affinités (oui, cela arrive...), restent à l'origine de tous les processus de création humaine, dont celui de la recherche. J'en profite donc pour saluer la venue du prochain congrès de l'ARIC à Rabat au Maroc, du 9 au 13 décembre 2013. Ce sera sûrement un nouvel événement propice à l'établissement de ces relations créatrices de sens.

Bonne lecture!

\section{Rattachement de l'auteur}

${ }^{1}$ Université Laval, Québec, Canada

\section{Correspondance}

alterstice@gmail.com

\section{Pour citer cet article :}

Leanza, Y. (2013). Fabriquer un numéro : des relations et du temps [Éditorial]. Alterstice, 3(1), 1-2. 